\title{
Movilización del conocimiento: aportes para los estudios sociales de la salud
}

\author{
Knowledge Mobilization: Contributions for \\ Social Studies in Health
}

\section{Mobilização do conhecimento: aportes para os estudos sociais da saúde}

\author{
Soledad Rojas Rajs, PhD ${ }^{1 *}$ \\ José Miguel Natera, $\mathrm{PhD}^{1}$
}

Recibido: 10 de septiembre de 2018 - Aceptado: 08 de abril de 2019

Doi: http://dx.doi.org/10.12804/revistas.urosario.edu.co/revsalud/a.8369

Para citar este artículo: Rojas Rajs S, Natera JM. Movilización del conocimiento: aportes para los estudios sociales de la salud. Rev Cienc Salud. 2019:17(3):111-131. Doi: http://dx.doi.org/10.12804/revistas.urosario.edu.co/revsalud/a.8369

\section{Resumen}

Introducción: las brechas entre la investigación en salud y su aplicación práctica constituyen un objeto de estudio y un campo de intervención en la esfera sanitaria. La traducción y uso del conocimiento basado en investigación se consideran cruciales para mejorar la salud pública y los sistemas de salud. Desarrollo: el objetivo de este trabajo es revisar y comparar los enfoques conceptuales investigación traslacional (translational research), traslación del conocimiento (knowledge translation) y movilización del conocimiento (knowledge mobilization). Estos enfoques se han desarrollado teórica e institucionalmente en países con fuertes sistemas de investigación en salud, como Estados Unidos, Canadá y Reino Unido, mientras que en Latinoamérica han sido poco trabajados. Se identifican sus objetivos, concepción de los procesos de transferencia de conocimiento, principales aportes y limitaciones. El enfoque movilización del conocimiento en salud se destaca como una propuesta más amplia, que aborda el carácter social de la producción, transferencia y uso del conocimiento. Conclusiones: el enfoque de movilización de conocimiento puede ser un marco útil para el estudio y sistematización de experiencias sobre el uso del conocimiento en salud en la región latinoamericana, a fin de fortalecer los sistemas nacionales de investigación en salud.

Palabras clave: movilización del conocimiento, investigación traslacional, traslación del conocimiento, investigación en salud.

\section{Abstract}

Introduction: The gaps between health research and its practical application constitute an object of study and a field of intervention in the health sphere. The translation and use of research-based knowledge are considered crucial to improve public health and health systems. Development: The aim of this paper is to review and compare the conceptual approaches translational research, knowledge translation, and knowledge mobilization. These approaches have been theoretically and institutionally developed

Conacyt - Universidad Autónoma Metropolitana Xochimilco (México).

Autora de correspondencia: srojasrajs@gmail.com 
in countries with strong health research systems such as the United States, Canada, and the United Kingdom, while in Latin America, they have not been sufficiently studied. To analyze the knowledge transfer processes conception and their main contributions and limitations. The knowledge mobilization approach stands out as a wider framework, which includes the social nature of the production, transfer, and use of knowledge. Conclusions: The knowledge mobilization approach is a suitable framework for studying and systematizing experiences on the use of health knowledge in Latin America, aiming at strengthening National Health Research Systems.

Keywords: Knowledge mobilization, translational research, knowledge translation, health research.

\section{Resumo}

Introdução: as brechas entre a pesquisa em saúde e a sua aplicação prática constituem um objeto de estudo e um campo de intervenção na esfera sanitária. A tradução e uso do conhecimento baseado em pesquisa consideram-se cruciais para melhorar a saúde pública e os sistemas de saúde. Desenvolvimento: o objetivo deste trabalho é revisar e comparar os enfoques conceituais pesquisa translacional (translational research), translação do conhecimento (knowledge translation) e mobilização do conhecimento (knowledge mobilization). Estes enfoques se têm desenvolvidos teórica e institucionalmente em países com fortes sistemas de pesquisa em saúde, como os Estados Unidos, a Canadá, e o Reino Unido enquanto na Latino-América têm sido pouco trabalhados. Se identificam seus objetivos, concepção dos processos de transferência de conhecimento, principais aportes e limitações. O enfoque mobilização do conhecimento em saúde destaca-se como uma proposta mais ampla, que aborda o carácter social da produção, transferência e uso do conhecimento. Conclusões: o enfoque de mobilização do conhecimento pode ser um marco útil para o estudo e sistematização de experiências sobre o uso do conhecimento em saúde na região latino-americana, com o objetivo de fortalecer os sistemas nacionais de pesquisa em saúde.

Palavras-chave: mobilização do conhecimento, pesquisa translacional, translação do conhecimento, pesquisa em saúde.

\section{Introducción}

Transformar el conocimiento científico sanitario en acciones e intervenciones es un factor clave para mejorar la salud de los colectivos humanos, atender los problemas nacionales de salud y alcanzar mayor grado de desarrollo humano (1). Por ello, reducir o sortear las brechas entre la investigación en salud y su aplicación práctica constituye un objeto de estudio y un campo de intervención.

En el mundo se ha impulsado el desarrollo de sistemas nacionales de investigación en salud (sNis) (1-3), con el soporte institucional de políticas y prácticas que promueven el aprovechamiento y uso del conocimiento basado en investigación. Sin embargo, el grado de desarrollo de estos sistemas es diverso y, en general, asimétrico (1, 4). Países como Reino Unido, Estados Unidos o Canadá - entre otros- tienen un alto grado de desarrollo institucional en torno a la investigación en salud, mientras en los países latinoamericanos estos sistemas aún son emergentes $(4,5)$. Adicionalmente, en América Latina existen grandes diferencias entre países, en términos de capacidades científicas y tecnológicas, y en el desarrollo de políticas, programas y acciones específicas para el uso de la investigación en salud. 
En tal sentido, el objetivo de este trabajo es revisar y analizar el desarrollo teórico e institucional de países con fuertes sistemas nacionales de investigación en salud, sobre cómo trasladar conocimiento científico sanitario, puesto que son marcos específicos para el campo de la salud. Se trata específicamente de analizar la adopción institucional de propuestas de investigación traslacional (гт) en Estados Unidos (6-8); traducción o traslación del conocimiento (тс) en Canadá (9-11); y la más reciente propuesta de movilización del conocimiento (Mc) de Canadá y Reino Unido. Estos enfoques se han desarrollado en términos teóricos y en las políticas e instituciones del sistema de salud, y su discusión y estudio se ha abordado en forma creciente en la literatura especializada (12).

La primera sección de este documento reseña brevemente los términos del debate sobre conocimiento para una mejor salud de fines de los años noventa y la década de 2000. A continuación, se revisa cada uno de los enfoques sobre conocimiento seleccionados, con base en sus textos fundacionales y su adopción institucional. La siguiente sección compara sus características conceptuales, rasgos distintivos y esboza los anclajes institucionales de los modelos revisados. Esta comparación muestra que la movilización de conocimiento (мc) es un marco más amplio, que permite recuperar los aportes de los otros dos e incluir el aporte teórico de las ciencias sociales, el cual reconoce que las actividades científicas de la esfera sanitaria ocurren en el marco de relaciones asimétricas y de poder, en contextos determinados, y que tienen dimensiones culturales, políticas y económicas. Se discute que a partir del marco sobre мс se pueden abordar las especificidades tanto del campo sanitario como de los contextos nacionales y regionales latinoamericanos, que comparten algunas características comunes, como la desigualdad social, las inequidades sanitarias y las asimetrías en el desarrollo de sNis.

Finalmente, se concluye sobre la importancia de estudiar y sistematizar experiencias latinoamericanas acerca del uso del conocimiento en salud, que permitan caracterizar y tipificar procesos regionales o nacionales. Se asume que este ejercicio tiene alcances limitados, pues no sistematiza directamente estas experiencias. Su aporte se centra en delinear una discusión pendiente para la región, donde no contamos con marcos analíticos propios sobre producción, movilización y uso de conocimiento en salud, mas se reconoce la necesidad de una discusión multidisciplinar para refinar esta propuesta analítica. 


\section{Desarrollo}

\section{Antecedentes: conocimiento para una mejor salud}

El desarrollo de conocimiento científico en el campo de la salud y el impulso a su utilización práctica ha sido abordado desde distintas teorías y marcos analíticos que no siempre coinciden o confluyen (10,13-16). Aun así, existe alto grado de consenso sobre el gran valor del conocimiento basado en investigación científica para mejorar la situación de salud de individuos, poblaciones, países y regiones (1).

Desde los años noventa emerge la reflexión sobre la gestión del conocimiento en salud y su valor e importancia estratégica para el desarrollo económico. A partir del año 2000, la transferencia de conocimientos derivados de investigación se definió como un objetivo deseable para la investigación en salud en el mundo, en la Conferencia Internacional sobre Investigación en Salud para el Desarrollo en Bangkok $(2,17)$. Ahí, se planteó que el conocimiento es un "bien público global" (2). Diez años antes, en 1990, la misma conferencia había destacado la importancia de la investigación sanitaria nacional sobre salud y la necesidad de desarrollar snis con el objetivo de mejorar la situación sanitaria de los países. En la Conferencia de Bangkok se planteó que una de las cuatro funciones principales de los sNis debía ser la gestión del conocimiento, entendida como producción y aplicación de este; no obstante, se observaron desiguales capacidades de investigación y desempeño, así como diferentes obstáculos para realizar exitosamente esta gestión entre los países miembros de la conferencia $(1,2,18)$.

En la primera década del siglo xxi transferir conocimiento en salud comenzó a delinearse como un campo de intervención especializado. Desde 2003 la Organización Mundial de la Salud fundó el departamento de gestión del conocimiento, se publicaron reportes y se organizaron conferencias internacionales $(1,18,19)$. En la Cumbre Ministerial sobre Investigación en Salud de la Ciudad de México, en 2004, 52 países firmaron una declaración para fortalecer los sistemas de salud, incluidos los snIs, a fin de conseguir el logro de "conocimiento para una mejor salud" y superar las brechas entre conocimiento y acción. Las brechas comenzaron a caracterizarse: distancias disciplinarias, asimetrías en capacidades científicas y tecnológicas, falta de recursos financieros, limitadas o ausentes políticas institucionales que fomentasen la vinculación, creación de canales e interacción entre actores relevantes (19). Quedó claro que la investigación en salud, por sí misma, no aseguraba el uso del conocimiento.

A partir del año 2006, el tema cobró una importancia sin precedentes y se efectuaron fuertes inversiones económicas en países como Estados Unidos y Reino Unido, y la movilización y uso del conocimiento empezó a ser tema de estrategias nacionales, tanto por los efectos esperados en la salud como por el impulso al crecimiento económico de las innovaciones médicas. Se crearon fondos específicos, instituciones de investigación, y de forma significativa 
comenzaron a aumentar las publicaciones científicas sobre transferencia del conocimiento en salud, así como nuevas revistas científicas. Si al inicio de la década de 2000 existían solo algunas revistas, como el Journal of Translational Medicine (uk, 2003) o Translational Medicine (USA, 2005), para el año 2011 existían al menos 27 publicaciones que incluían la palabra 'traslacional' indexadas en el National Center for Biotechnology Information (NCBI) de revistas médicas (12, 20); el $67 \%$ de estas revistas fueron creadas después de 2008. Para el año 2015, en este mismo índice aparecen más de 80 revistas traslacionales.

Esta tendencia creciente en las publicaciones se ha mantenido en Reino Unido, Canadá, Estados Unidos y países europeos como Noruega, Suecia y Holanda, y también en países como Japón. En cambio, en América Latina no se incrementó sustancialmente la producción sobre los enfoques traslacionales o el enfoque de movilización del conocimiento, aunque aparecieron algunos trabajos a partir del año 2006 (21-24). Un suceso similar acontece con la propuesta de movilización del conocimiento a partir del año 2008, en cuanto a incremento de publicaciones, aunque con mayor fuerza en el campo de la educación (25).

\section{Investigación traslacional: centrada en las brechas por áreas de conocimiento}

El concepto investigación traslacional (тт) comenzó a utilizarse en los años noventa en la investigación sobre cáncer en Estados Unidos para designar los procedimientos de investigación que permitirían lograr la aplicación clínica de los avances biomédicos $(26,27)$. Es una estrategia incluida en el programa de reingeniería de la investigación clínica que presentaron los Institutos Nacionales de Salud en $2003(28,29)$. La iт se planteó como una vía para mejorar la velocidad de adaptación de los conocimientos a aplicaciones prácticas, siguiendo el precepto bench-to-bedside (del laboratorio al paciente) y se puede identificar como un modelo en etapas (15). Se concibió inicialmente la existencia de dos bloques traslacionales (figura 1), es decir, dos conjuntos de obstáculos que deben superarse en las etapas de desarrollo de productos y servicios sanitarios, ya que dificultan la transferencia y la aplicación del conocimiento (6).

El primer bloque, T1, destaca los problemas para llevar los hallazgos de laboratorio a la investigación en humanos, en estadios preclínicos.

El bloque T2 se refiere a los obstáculos para lograr la adopción de intervenciones o desarrollos ya probados en humanos en la práctica clínica. Los bloques traslacionales sintetizan las dificultades para conseguir estos pasos: la escasez de recursos humanos y materiales, la falta de incentivos o dificultades derivadas de los marcos regulatorios, entre otros.

Sin embargo, se ha señalado la insuficiencia del esquema T1 y T2 para abordar la complejidad del campo de la salud $(12,30)$. Particularmente, se cuestiona que en el esquema de fases se identifique a la investigación básica como el único origen de los posibles desarrollos o intervenciones en salud. Se objeta que la iт esté centrada en el desarrollo de productos farmacéuticos, pues 


\section{Bloques traslacionales}

Falta de participantes dispuestos

Carga regulatoria

Infraestructura fragmentada

Bases de datos incompatibles

Falta de investigadores calificados
Desincentivos en las carreras Limitaciones en la práctica médica Altos costos de la investigación Falta de financiamiento
Investigación básica

biomédica
Traslación de la ciencia básica a los estudios en humanos

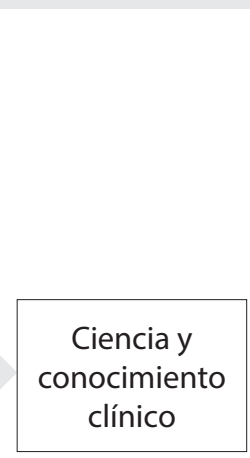

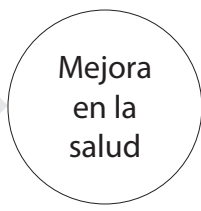

Continuo de la investigación clínica

Figura 1. Bloques traslacionales en el continuum de la investigación clínica, retos centrales frente a la empresa nacional de investigación clínica (6)

Fuente: Sung NS, Crowley Jr. WF, Genel M, Salber P, Sandy L, Sherwood LM, et al. Central challenges facing the national clinical research enterprise. J Am Med Assoc. 2003;289(10):1278-87 (6).

inicialmente no reconoció la importancia de la investigación en salud pública (31). Y se cuestiona también que los fondos para investigación se concentran en los tipos básica y biomédica básica, en detrimento de la investigación en sistemas de salud, indispensable para la integración del conocimiento a las prácticas sanitarias (30). A partir de estas críticas y cuestionamientos, en la literatura especializada se elaboraron nuevas propuestas, que plantean más fases de investigación y relaciones bidireccionales entre metodologías de investigación y fases de producción de conocimiento (32); no obstante, no se ha logrado romper la linealidad ciencia básica-clínica-práctica-sistema de salud, lo que define las áreas privilegiadas de políticas públicas de rт.

Casi una década después de publicar sobre los bloques traslacionales de la iт, en Estados Unidos se fundó en 2012 el Centro Nacional para el Avance de las Ciencias Traslacionales, un nuevo instituto nacional de salud. Su misión es catalizar métodos y tecnologías innovadoras que mejoren aspectos diagnósticos y terapéuticos. A partir del concepto 'ciencias traslacionales', buscan englobar distintas áreas de conocimiento en salud y presentar relaciones no lineales entre etapas traslacionales. Esta nueva visión está orientada a promover el proceso de traducción en todas las diferentes etapas de las actividades de investigación en salud, sin embargo, el énfasis en la investigación básica permanece. Su aporte fundamental es que señala que en el propio campo sanitario la diversidad de áreas del conocimiento y disciplinas requiere establecer entre sí puentes de comunicación y traducción, con apoyo en estímulos y regulaciones. 


\section{Traslación del conocimiento: centrado en el proceso de aplicación del conocimiento}

Traslación (o traducción) del conocimiento (Tc) es un concepto acuñado en el año 2000 en Canadá por los Institutos Canadienses de Investigación en Salud (CIHR), principal agencia gubernamental de financiamiento a la investigación sanitaria (33). La тс se definió como un modelo expresamente diseñado como alternativa a la linealidad y unidireccionalidad asociada a modelos tradicionales de transferencia de conocimiento $(34,35)$. El desarrollo conceptual de la traducción del conocimiento en Canadá se nutrió del enfoque de medicina basada en evidencia (36), también de origen canadiense.

Al igual que en el caso de la rт, uno de los argumentos principales para desarrollar modelos de тс es lograr la aplicación y uso del conocimiento, así como acortar los largos períodos para el desarrollo y adopción de conocimientos científicos en salud (10,37). También se busca vincularlos con el sistema de salud y las acciones de salud pública. Straus, Tetroe y Graham definen la тc como un proceso dinámico, centrado en la aplicación del conocimiento, que incluye procesos de comunicación (síntesis, difusión e intercambio de conocimientos) y procesos de aplicación en servicios, productos, y en el sistema de salud (9).

Un primer modelo conceptual propuesto por los cirr fue el "modelo de traslación de conocimiento global” (figura 2), que representa seis etapas en los procesos de investigación, en las que se fomentan las interacciones entre los usuarios del conocimiento o los socios de la investigación (38).
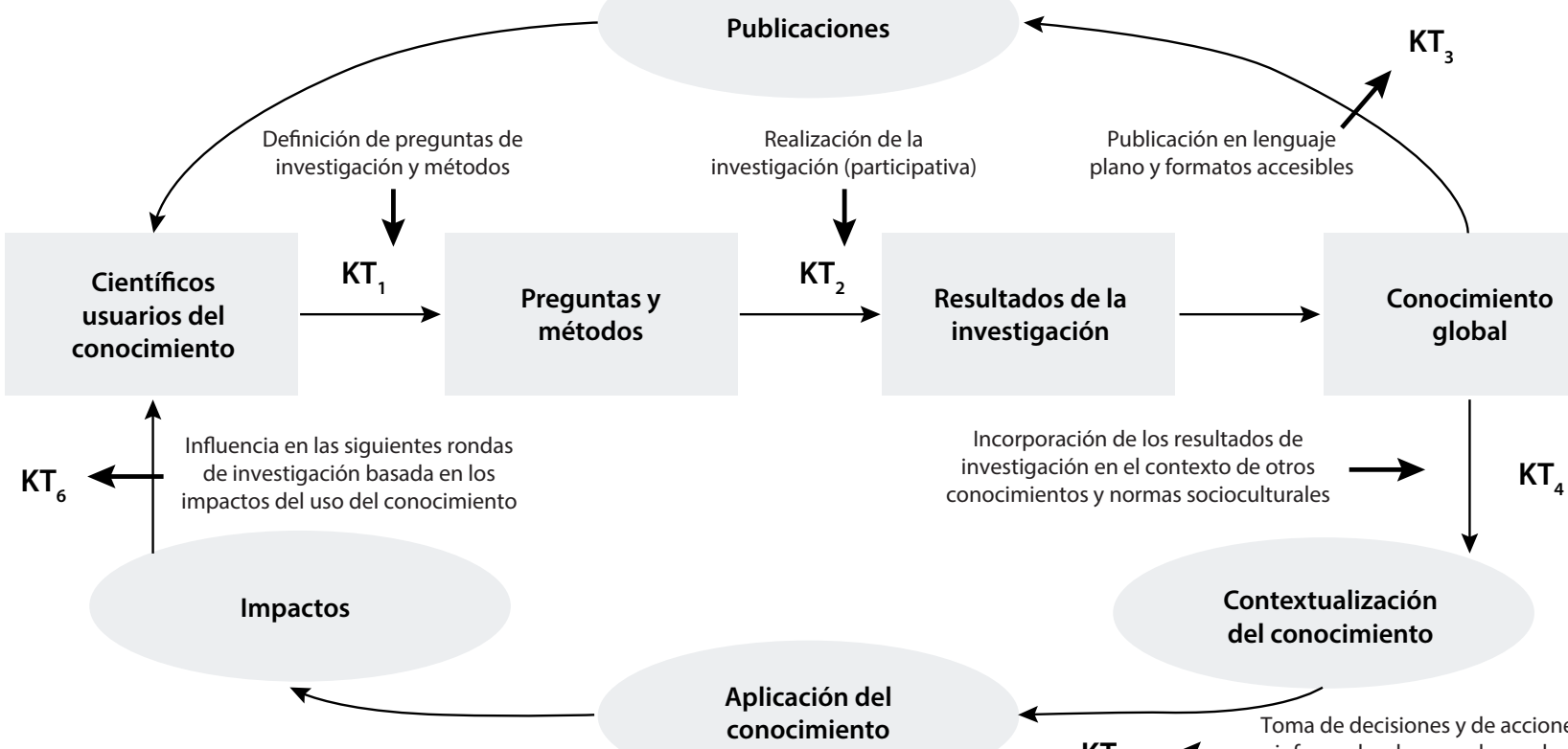

Contextualización del conocimiento

Aplicación del conocimiento

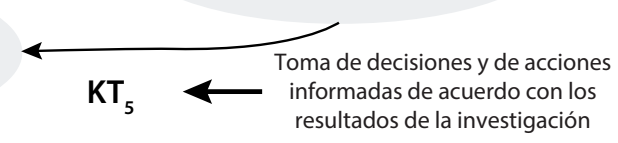

Figura 2. Modelo de Tc global y ciclo de investigación de la cIHR: seis oportunidades para facilitar la traslación del conocimiento (38)

Fuente: Sung NS, Crowley Jr. WF, Genel M, Salber P, Sandy L, Sherwood LM, et al. Central challenges facing the national clinical research enterprise. J Am Med Assoc. 2003;289(10):1278-87 (6). 
Los seis pasos del modelo global de traslación del conocimiento son los siguientes:

1. TC1: definición de preguntas y métodos de investigación.

2. TC2: realización de investigaciones (participativas).

3. TC3: publicación en lenguaje sencillo y formatos accesibles.

4. TC4: ubicación de hallazgos de investigación en el contexto de otros conocimientos y normas socioculturales.

5. TC5: tomar decisiones y medidas informadas por los resultados de la investigación.

6. TC6: influir en las siguientes rondas de investigación basadas en los impactos del uso del conocimiento.

En este modelo, el punto de partida y llegada son TC1 y TC6, respectivamente, y se incluyen como actores: investigadores y usuarios del conocimiento.

La perspectiva de Tc destaca el carácter social y cultural de los procesos, ya que implican interacciones humanas, involucran investigadores y conocimiento de usuarios finales, y están mediados por factores culturales u otros contextos, es decir, resalta la perspectiva de actores e interacciones, en particular mostrando que las interacciones entre los actores y los responsables de la toma de decisiones son fundamentales para el éxito de los procesos de traducción (39). Los usuarios de conocimiento son reconocidos como actores con cierto grado de influencia en los procesos de traducción, no son solo receptores pasivos de productos o ideas. Este modelo también toma en cuenta que múltiples actores participan en la producción y el uso del conocimiento de la salud (40). Comprender la traducción del conocimiento en el marco de las relaciones sociales ha favorecido el establecimiento de elaboraciones teóricas más complejas sobre los procesos de traducción. Arreglos entre agentes, coproducción de conocimiento y la noción de que existen resistencias y problemas sociales para la aplicación de conocimiento han sido integrados como temas en la agenda de salud en Canadá gracias al modelo de тс $(41,42)$. No obstante, la perspectiva no es sistémica, sino que representa una ruta crítica que modela los proyectos de investigación, lo que se muestra en el modelo de conocimiento-hacia-la-acción (10) (figura 3).

El modelo se ocupa fundamentalmente del proceso de implementación: define ciclos de acción continuos que se originan a partir de síntesis de conocimiento científico. En pasos subsecuentes, se trata de identificar problemas; adaptar el conocimiento a los contextos locales; evaluar barreras y facilitadores para el uso del conocimiento; seleccionar medidas específicas para la implementación; monitorear y evaluar el uso del conocimiento y sus resultados, y promover el uso continuo del conocimiento en el tiempo. El modelo recupera teóricamente la comprensión del contexto y la participación de usuarios del conocimiento, logrando así adaptaciones locales (38). 


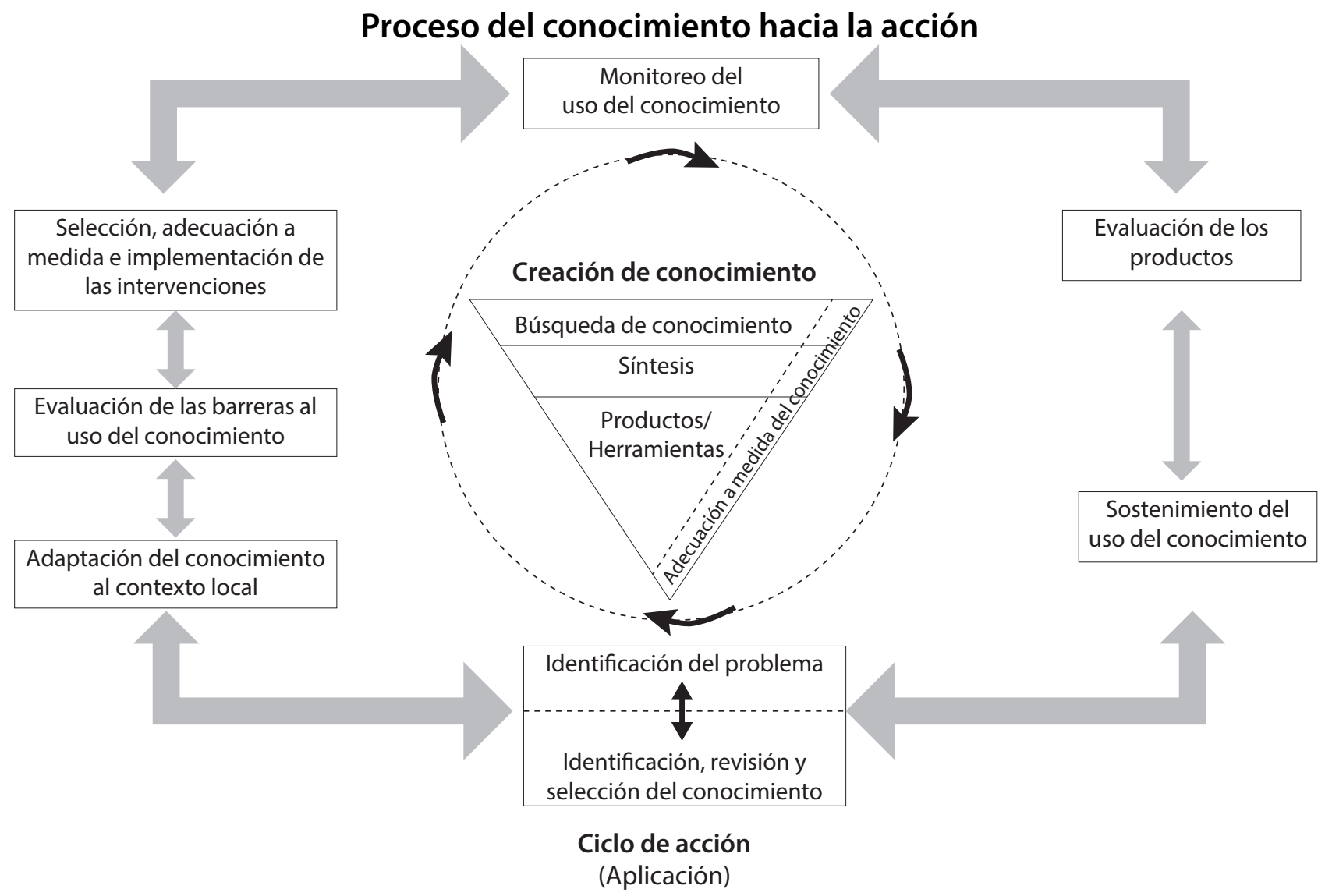

Figura 3. Proceso de conocimiento-hacia-la-acción de los Institutos Canadienses de Investigación en Salud (cirR) Fuente: elaboración propia basada en Graham I, Logan J, Harrison MB, Straus SE, Tetroe J, Caswell W, et al. Lost in knowledge map: time for a map? J Contin Educ Health Prof. 2006;26(1):13-24 (10).

Aunque la propuesta de conocimiento-para-la-acción es conceptualmente rica, depende en gran medida de un fuerte apoyo institucional, como el proporcionado por los cirr, que cuentan con fondos específicos para la implementación del modelo. Aun así, tiene dificultades prácticas. En una revisión sistemática reciente, se encontró que, de los 1057 artículos referidos a este modelo entre 2006 y 2012, solo 10 (1\%) estaban relacionados con experiencias de investigación prácticas que integraron todo el modelo, desde la fase de creación de conocimiento hasta el ciclo de acción (43); 8 de estos artículos eran canadienses, por lo que su extrapolación empírica no parece ser fácil, especialmente fuera de un esquema institucional que soporta los requerimientos del modelo.

\section{Movilización del conocimiento: un diálogo con las ciencias sociales}

Un concepto propuesto inicialmente desde las ciencias sociales y no desde las ciencias de la salud es la movilización del conocimiento (мс) (44). El término fue impulsado por el Consejo de Investigación de las Ciencias Sociales y Humanidades de Canadá (ssHrc). Por ello, está centrado en el carácter social de los procesos mc: describe actividades que conforman flujos 
de conocimiento basado en investigación, en y entre actores académicos y hacia la sociedad. Está enfocado en los procesos de comunicación y circulación del conocimiento entre actores, como condición indispensable para su utilización y puesta en práctica, que están situados en contextos específicos y características locales.

En términos de proceso y direccionalidad, la mc se plantea como un flujo recíproco y complementario de conocimientos basados en investigación, que circulan entre investigadores, agentes de conocimiento y usuarios del conocimiento. Esta circulación del conocimiento es positiva para quienes participan y puede conducir a beneficios concretos, a la transformación de conocimiento en prácticas.

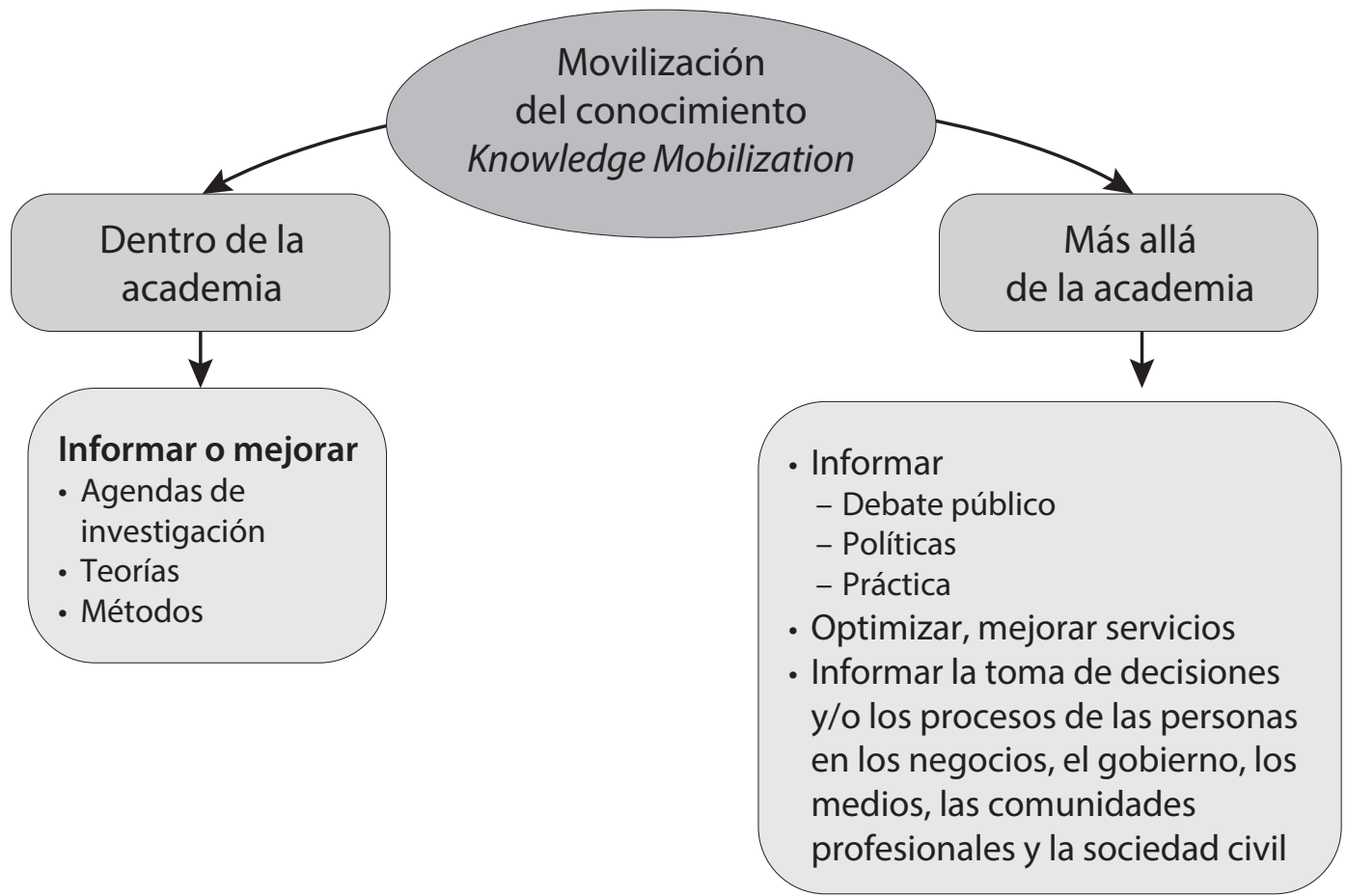

Figura 4. Movilización del conocimiento, SSHRC-CRSH, Canadá

Fuente: elaboración de los autores basada en la definición del Consejo de Investigación en Ciencias Sociales y Humanidades de Canadá (2018). Disponible en: http://www.sshrc-crsh.gc.ca

En el campo de la salud en Reino Unido, Davies, Powell y Nutley han conceptualizado la Mc como todas aquellas actividades encaminadas a confirmar y comunicar conocimientos basados en investigación científica en el contexto del sistema de atención a la salud, o bien dentro de las organizaciones que trabajan en atención a la salud y provisión de servicios sanitarios (14). Como propuesta, está enfocada principalmente en llevar el conocimiento basado en investigación a las políticas o a las prácticas de atención a la salud.

La мc se elaboró a partir de los procesos de discusión en Canadá sobre conocimiento, incluidos los del campo sanitario, especialmente sobre тс (44). No obstante, al ser resultado de un trabajo interdisciplinario entre ciencias sociales y ciencias de la salud, el concepto permite una comprensión más amplia de los procesos de producción, circulación y uso del 
conocimiento que los enfoques traslacionales. Por ejemplo, enfatiza que el conocimiento se construye socialmente y que existen múltiples formas de usarlo, lo que influye en cuán directo, oportuno o efectivo puede resultar este uso. En este sentido, tiene cercanía con las propuestas latinoamericanas sobre apropiación social del conocimiento, principalmente la mirada sobre apropiación “fuerte”, que, si bien no es específica del campo sanitario, destaca la importancia de la producción interdisciplinaria de conocimiento y de las interacciones entre los distintos actores que participan de la mc, con el propósito de fomentar el uso social de la investigación científica y la democratización de la ciencia $(45,46)$. En el marco de los estudios sociales de la ciencia y la tecnología en la región latinoamericana, se observan los efectos políticos, culturales y económicos del conocimiento científico y la innovación tecnológica, aunque no se han elaborado marcos específicos para los problemas sanitarios (45). En este sentido, la мc es una propuesta que puede articularse con el trabajo previo en la región.

La propuesta de mc en salud considera que los conocimientos basados en investigación tienen múltiples usos, de entre los cuales los principales son: a) el uso instrumental o directo, b) el uso conceptual y c) el uso simbólico $(14,47)$. El uso instrumental del conocimiento se refiere a la aplicación en formas específicas y directas, y es más valorado desde la perspectiva de investigación y desarrollo (I+D). Sin embargo, el uso conceptual o simbólico del conocimiento también puede tener un efecto importante en el campo sanitario. Por ejemplo, el uso conceptual o indirecto influye en el mediano y largo plazo, puesto que puede producir una forma distinta de comprender la salud-enfermedad, o cambios de actitudes hacia riesgos 0 condiciones sanitarias. El uso conceptual del conocimiento basado en investigación puede influir en la significación social en salud, las representaciones sociales y las interpretaciones culturales de los fenómenos sanitarios. Por último, cuando se habla de uso simbólico del conocimiento, se refiere a la utilización política o persuasiva de la ciencia para sostener o legitimar posiciones. Este último uso, no menos relevante, puede favorecer el cambio o la resistencia al cambio, pues sirve como argumento para la toma de decisiones.

En este sentido, la mc se entiende como un proceso que ocurre en el marco de relaciones sociales existentes, en las que se producen interacciones y aprendizajes, que son procesos sociales situados y en diversa correlación de fuerzas. Por ello, tiene una dimensión política, pues implica una negociación entre actores en torno a los significados, encuadres y definiciones de los problemas. Esto, en el caso de los problemas de salud, tiene un fuerte sesgo disciplinario, por los escasos puentes de comunicación entre áreas de conocimiento. Se aborda así el problema de las relaciones de poder en la movilización del conocimiento.

En términos del proceso, el marco de mc plantea que el conocimiento por sí mismo no tiende a circular y que para que los flujos de conocimiento se produzcan es necesario implementar estrategias activas para la movilización, especialmente porque los flujos de conocimiento en la esfera sanitaria suelen ser lentos e intermitentes, o, incluso, inciertos (14). Para generar estrategias efectivas, se requiere definir qué conocimiento se quiere comunicar, 
a quién, con la colaboración de quién, de qué manera y para lograr qué objetivo. Esto significa considerar la naturaleza del conocimiento, cuál será su uso, así como la definición de audiencias y formatos para su movilización. Como parte de las estrategias de мc, se establece además la figura de mediadores del conocimiento (knowledge brokers), y la importancia de estimular la conectividad, es decir, las interacciones entre los actores que participan o se benefician de los flujos de conocimiento.

Con base en Davies, Powell y Nutley, los componentes conceptuales por considerar para la mc son los siguientes (14):

1. Uso del conocimiento

a) Diferentes tipos de conocimiento

b) Distintos usos del conocimiento

2. Audiencias
a) Agentes del conocimiento
b) Audiencias
c) Mediadores (knowledge brokers)
d) Tomadores de decisiones

3. Contextos (múltiples)

a) Interno:

- Estructuras

- Procesos organizacionales

- Culturas organizacionales

b) Externo:

- Clima político, social y cultural

- Estabilidad ambiental

- Comunicación interorganizacional

- Marco institucional y regulatorio

4. Flujos de conocimiento (creación activa)

a) Actividades académicas y de comunicación

b) Mejores formatos para мc (p. ej., mayor efectividad si se movilizan cuerpos de conocimiento que conocimientos individuales; formatos distintos para legos que para especialistas)

Con estas consideraciones, la mc permite integrar los temas centrales de los enfoques traslacionales, sobre la naturaleza del conocimiento (гт) y sobre los actores y sus contextos 
(тс). Y, aunque parte de una mirada más general, su aplicación estratégica puede ser más específica en tanto permite la planificación de la movilización del conocimiento. En Reino Unido y Canadá, las universidades e instituciones que han adoptado el término han desarrollado guías para la planificación estratégica.

Finalmente, desde la propuesta de mc, se reconoce que el conocimiento basado en investigación coexiste con conocimientos tácitos y epistemologías, o formas de significar culturalmente la vida y los procesos de salud-enfermedad. Los flujos de conocimiento son, por lo tanto, bidireccionales y susceptibles de retroalimentación.

\title{
Principales aportes y limitaciones de los enfoques conceptuales sobre conocimiento
}

\author{
En la tabla 1 se han sintetizado las características principales y los rasgos distintivos de cada \\ enfoque.
}

Tabla 1. Enfoque sobre conocimiento basado en investigación: investigación traslacional, traslación del conocimiento y movilización del conocimiento

\begin{tabular}{|c|c|c|c|}
\hline & Investigación traslacional & $\begin{array}{l}\text { Traslación del } \\
\text { conocimiento }\end{array}$ & $\begin{array}{l}\text { Movilización del } \\
\text { conocimiento }\end{array}$ \\
\hline Definición principal & $\begin{array}{l}\text { - Tomar la investigación bá- } \\
\text { sica o clínica para producir } \\
\text { innovación en entornos de } \\
\text { atención médica. Define la } \\
\text { investigación que involu- } \\
\text { cra investigación básica y } \\
\text { aplicada: es la interfaz en- } \\
\text { tre los dos extremos (48). }\end{array}$ & $\begin{array}{l}\text { Proceso dinámico y repe- } \\
\text { tido, que incluye la sínte- } \\
\text { sis, difusión, intercambio y } \\
\text { aplicación ética del conoci- } \\
\text { miento, para mejorar la sa- } \\
\text { lud, proporcionar servicios y } \\
\text { productos de salud más efec- } \\
\text { tivos y fortalecer el sistema } \\
\text { de atención a la salud (9). }\end{array}$ & $\begin{array}{l}\text { - Dar información basada en } \\
\text { investigación a la gente co- } \\
\text { rrecta, en el formato correc- } \\
\text { toy en el momento correcto } \\
\text { para influir en su toma de } \\
\text { decisiones. La Mc incluye } \\
\text { difusión, transferencia de } \\
\text { conocimiento y traducción } \\
\text { del conocimiento (44). }\end{array}$ \\
\hline Objetivo central & $\begin{array}{l}\text { Probar en humanos nuevas } \\
\text { estrategias terapéuticas de- } \\
\text { sarrolladas en laboratorio } \\
\text { (bench-to-bedside). }\end{array}$ & $\begin{array}{l}\text { - Aplicación del } \\
\text { conocimiento. }\end{array}$ & $\begin{array}{l}\text { - Comunicación y mediación } \\
\text { del conocimiento. }\end{array}$ \\
\hline $\begin{array}{l}\text { Modelo/concepción } \\
\text { del proceso }\end{array}$ & - Modelo lineal en etapas. & - Modelo por ciclos. & $\begin{array}{l}\text { - Modelo de flujos activos de } \\
\text { conocimiento. }\end{array}$ \\
\hline Punto de partida & $\begin{array}{l}\text { - Conocimientos fundamen- } \\
\text { tales, ciencias básicas. }\end{array}$ & $\begin{array}{l}\text { - Definición de problemas y } \\
\text { preguntas de investigación. }\end{array}$ & $\begin{array}{l}\text { - Definición de qué conoci- } \\
\text { miento se busca movilizar. }\end{array}$ \\
\hline Rasgo distintivo & $\begin{array}{l}\text { - Etapas o bloques traslacio- } \\
\text { nales (enfrentan brechas } \\
\text { entre áreas de conoci- } \\
\text { miento). }\end{array}$ & $\begin{array}{l}\text { - Proceso de traslación hacia } \\
\text { la acción (enfrenta obstácu- } \\
\text { los para la implementación } \\
\text { del conocimiento). }\end{array}$ & $\begin{array}{l}\text { - Flujos de conocimiento } \\
\text { (enfrentan barreras para } \\
\text { la circulación del conoci- } \\
\text { miento). }\end{array}$ \\
\hline
\end{tabular}

(Continúa) 
Investigación traslacional

- Destaca un importante problema en las ciencias de la salud: la fragmentación disciplinaria y la dificultad para compartir hallazgos entre áreas del conocimiento.

Principal aporte - Propone soluciones científicas: mejor formación de RR. HH. y equipos multidisciplinarios.
Traslación del conocimiento
Movilización del conocimiento
Describe ciclos de investigación y destaca la importancia de integrar a los beneficiarios del conocimiento.

- Propone coproducción de conocimiento, adaptación del conocimiento a contextos locales, monitoreo de la implementación del conocimiento.

- Hace explícita la importancia de pasar por procesos de adaptación del conocimiento (apropiación).
Reconoce el carácter social de los procesos de creación, movilización y uso del conocimiento.

- Integra la dimensión del poder como parte del análisis de contextos.

- Define estrategias activas de movilización del conocimiento.

- Define a mediadores del conocimiento como fundamentales para la construcción de capacidades para el uso del conocimiento.
- Es un modelo lineal que difícilmente puede adaptarse a la diversidad de áreas de conocimiento y metodologías que confluyen en las ciencias de la salud.

Principales limitaciones
- Está enfocado únicamente en el desarrollo de medicamentos o dispositivos médicos.

- Alta dependencia del desarrollo del sNis y la adopción de políticas para la ic.
- Como modelo de ciclos, es poco adaptable a situaciones de investigación que no adoptaron desde el inicio la intención de traducir conocimiento.

- Alta dependencia del desarrollo del snis y la adopción de políticas para la Tc.

- Requiere un gran soporte institucional y financiero.
Puede centrarse únicamente en la comunicación de información basada en información, sin llegar a la aplicación.

- Alta dependencia del desarrollo del sNis y la adopción de políticas para la mc.

Fuente: elaboración de los autores.

En esta matriz sintética se destaca que los modelos de los enfoques traslacionales conciben los procesos de transferencia de conocimiento como parte de las investigaciones y no como una actividad específica. En este sentido, representan etapas metodológicas, sea que se propongan con un modelo en etapas, como la investigación traslacional (IT); o bien como pasos en un ciclo de aplicación del conocimiento, en el caso de la Tc. En contraste, la propuesta de mc está centrada específicamente en cómo lograr su comunicación, difusión, adopción y apropiación, además de considerar algunas cuestiones más generales, como la especificidad de los contextos o el carácter político de las relaciones entre actores del conocimiento. En ese sentido, desde este trabajo se propone que la mc puede conducir a una descripción más específica de las actividades de salud y los flujos de conocimiento, en la que el contexto político y social, la complejidad organizacional y las relaciones de poder se aborden explícitamente.

La iт pone en el centro de la discusión el problema de la fragmentación disciplinaria de las ciencias de la salud, principalmente en relación con el traslado de los resultados de laboratorio a la práctica clínica. No obstante, la crítica principal para la тт es que su modelo lineal no reconoce que las relaciones e intercambios no son solo relaciones de laboratorio. 
Más allá de tender puentes entre la investigación básica, biomédica o clínica, es necesario comprender la naturaleza social de los procesos de transferencia de conocimiento. Estos son procesos y prácticas socialmente situados, cuyo sentido y dirección pueden ser mejor orientados y canalizados a partir de una comprensión profunda de los contextos, los usos y las relaciones e interacciones necesarias para fomentar la Mc. Adicionalmente, es necesaria una concepción dinámica sobre el tema, porque los cambios y transformaciones de los sistemas de salud y en los avances científicos implican la emergencia de nuevos actores y conducen a nuevas configuraciones y contextos tanto para el sistema de salud como para el de investigación en salud $(20,49)$. Estas configuraciones pueden ser especificadas y descritas como parte del contexto de la mc.

Esto no quiere decir que la Iт no sea un aporte para la aplicación del conocimiento, más bien define su marco y alcance específico al paso del laboratorio a la clínica. En contraste, el concepto y marco de тс es conceptualmente más amplio e inclusivo, tiene flexibilidad para aplicarse según diversos tipos de investigación en salud y puede referirse a una amplia gama de conocimientos en salud: desde las investigaciones clínicas y biomédicas, hasta la salud pública y la investigación sobre sistemas de salud. Al definir ciclos ideales para la aplicación del conocimiento, reconoce en todo momento a sus usuarios, destacando la importancia de integrar a estos usuarios o beneficiarios del conocimiento en los procesos de investigación. Introduce también, reconociendo que se trata de procesos socialmente situados, el concepto de coproducción de conocimiento, que alude a la participación de los usuarios del conocimiento en su proceso de generación. Esta es una condición favorable para la adopción y apropiación de nuevas técnicas o tecnologías en salud, conceptos, prácticas y políticas. La TC apunta a fomentar la circulación del conocimiento en una configuración no lineal que distribuye las fuentes de conocimiento durante un proceso cíclico, sin embargo, es claro que requiere un soporte en recursos financieros y políticas y programas gubernamentales muy fuerte.

El enfoque sobre Mc, al ser más amplio, permite englobar la Iт y la тc, y formular estrategias específicas de movilización del conocimiento dentro de estos modelos, aportando elementos adicionales: una mirada más profunda al contexto, la inclusión de la figura de los mediadores de conocimiento y la planificación de acciones de comunicación y vinculación que promuevan activamente la circulación de los flujos de conocimiento. Uno de sus aportes principales es que permite un diálogo con las ciencias sociales, en especial con aquellos campos de conocimiento que abordan la cuestión del uso social de la ciencia y la tecnología: los estudios sociales de la ciencia y los estudios sociales en salud.

Un aspecto relevante de тс, гт у мс es que solo pueden desarrollarse si se conciben y llevan a la práctica en el marco de la investigación nacional en salud, es decir, dependen de la fortaleza de los sNis (50-52). Es un obstáculo por considerar para la región latinoamericana, pues, aun en las mejores condiciones institucionales, no es sencillo lograr la aplicación 
del conocimiento (53). No obstante, los procesos se facilitan si existe un sNis formalmente estructurado y articulado, con roles definidos para sus actores y políticas definidas sobre investigación en salud y sus prioridades (4). Las instituciones formales juegan un papel fundamental en la determinación de la aplicación del conocimiento.

\section{Conclusiones}

Temos revisado los enfoques de investigación traslacional, traslación del conocimiento Ly movilización del conocimiento. El primero de ellos, Iт, busca cerrar las brechas de conocimiento entre las actividades de salud, utilizando un modelo lineal que va desde la ciencia básica hasta las aplicaciones de conocimiento en pacientes, pero su aporte fundamental es que muestra las enormes dificultades para integrar el conocimiento en salud, que se genera desde diversas disciplinas y áreas de la ciencia, las cuales tienen un alto grado de fragmentación.

El segundo, traducción del conocimiento, enfatiza la importancia de considerar que el conocimiento será utilizado por usuarios concretos en contextos específicos, por lo que busca integrar a los procesos de investigación la participación de los interesados y contempla la producción conjunta o coproducción de conocimiento, lo que favorece los procesos de adopción y apropiación. En este sentido, la тc es una propuesta más completa. Su limitación es la alta dependencia a un sistema institucional fuerte que la soporte y que su propuesta no define específicamente cuáles son las estrategias y actividades concretas que implica trasladar el conocimiento hacia la acción.

Desde este trabajo, consideramos relevante recuperar el enfoque de мс para la discusión latinoamericana. Por un lado, la complejidad de las actividades de salud es tan alta que requiere enfoques interdisciplinarios. Al integrar la mirada de las ciencias sociales con la propuesta de movilización del conocimiento es posible una mayor comprensión de las situaciones concretas en las que circula el conocimiento. Por otro, un enfoque que reconozca el carácter político de los procesos de movilización del conocimiento sanitario y las relaciones de poder que surgen entre agentes de conocimiento (universidades, instituciones del sector salud, comunidad científica nacional e internacional, etc.), así como sus asimetrías, permite recuperar más fielmente las condiciones concretas de los sistemas de salud en América Latina y su avance en investigación en salud.

El desarrollo conceptual en Latinoamérica de este tipo de enfoques sobre el conocimiento, su uso y movilización no ha sido específico para el campo sanitario, por ello, es fundamental avanzar en la discusión sobre las necesidades de mc, al igual que sobre las características de los países y sus problemas de salud. Esto implica priorizar cuáles son los principales problemas nacionales que se deben atender con conocimiento y cuáles las principales brechas y obstáculos para su movilización. 
Las formas específicas en que se significan los conceptos en el marco de los sistemas de salud, las políticas de salud y las de ciencia, tecnología e innovación en distintas experiencias nacionales, muestran que los contextos conforman escenarios concretos y particularidades -institucionales, normativas, económicas, políticas y culturales- que influyen en el nivel de consenso sobre transferencia de conocimiento y que pueden facilitar u obstaculizar los flujos de conocimiento y su puesta en práctica $(4,54)$. En este sentido, dos cuestiones resultan de gran importancia para facilitar la vinculación entre conocimiento basado en investigación y políticas y prácticas en salud: concebir los procesos del conocimiento en un marco institucional y generar aproximaciones basadas en la especificidad de estos marcos institucionales, que a su vez se relacionan con problemas de salud determinados y una configuración de actores que requiere detallarse según el entorno nacional.

En América Latina, aun cuando los sistemas de investigación en salud y las actividades de ciencia y tecnología han logrado mayor desarrollo en el tiempo, aún no contamos con marcos analíticos propios y específicos que describan o fomenten procesos de movilización e implementación del conocimiento sanitario basado en investigación. Por ello, es esencial abordar los conceptos y marcos que pueden ayudar al análisis y sistematización de las experiencias concretas con el conocimiento basado en investigación en el campo sanitario.

Así como se requiere fortalecer cada uno de los sNis de la región, el aprendizaje sobre cómo mejorar la тс en el campo de la salud requiere ser impulsado con la misma fuerza en los distintos tipos de investigación en salud. La tendencia a concentrar los recursos para la investigación en la investigación básica y biomédica no contribuye a desarrollar más ampliamente las actividades de mc, de carácter social y que requieren miradas interdisciplinarias (30,55).

Fortalecer el sistema nacional de innovación definiendo más formalmente las estructuras de los sistemas de salud puede tener un importante efecto en uno de los principales problemas que busca mitigar la тс y la iт: los grandes lapsos que desfasan los logros científicos de su aplicación. Estos pueden intensificarse en condiciones generales adversas para la aplicación de los conocimientos basados en investigación, pero, en contraparte, pueden mejorar a partir de mecanismos planificados e incentivos para la movilización y uso del conocimiento.

Mejores conexiones entre la investigación y la evidencia y las políticas y prácticas de salud son tareas pendientes para afrontar el panorama epidemiológico de la región, en donde confluyen los problemas de salud derivados de la transición epidemiológica, como las enfermedades crónicas; con las enfermedades carenciales; y la violencia, en un escenario de grandes desigualdades sociales y sanitarias. Un reto para el futuro será analizar la validez de estos enfoques conceptuales, con base en referentes empíricos concretos, que permitan adecuarse a los contextos locales y contribuir así a la solución de los problemas nacionales en salud. Esta tarea corresponderá a los estudios sociales sobre salud y a los estudios sociales de la ciencia y la tecnología. 


\title{
Contribución de los autores
}

\author{
mbos autores participaron en la planeación del artículo, desarrollo y aprobación \\ Afinal de esta versión.
}

\section{Conflicto de intereses}

Ninguno declarado

\section{Fuentes de financiación}

Esta investigación ha sido realizada con el apoyo del Conacyt a través del proyecto de la diabetes”, financiado por el Fondo de Ciencia Básica (CB2015-258387).

\section{Referencias}

1. World Health Organization. World report on knowledge for a better health. Geneva: wHо; 2004.

2. Pang T, Pablos-Mendez A, IJsselmuiden C. From Bangkok to Mexico: towards a framework for turning knowledge into action to improve health systems. Bull World Health Organ. 2004;82(10):719-810.

3. Hanney SR, González-Block MA. Building health research systems to achieve better health. Health Res Policy Syst. 2006;4:10.

4. Alger J, Becerra-Posada F, Kennedy A, Martinelli E, Cuervo LG, Al E. Sistemas nacionales de investigación para la salud en América Latina: una revisión de 14 países. Pan Am J Public Heal. 2009;26(5):447-57.

5. Dutrénit G, Capdevielle M, Corona JM, Punchet M, Santiago F, Vera-Cruz A. El Sistema Nacional de Innovación Mexicano: instituciones, políticas, desempeño y desafíos. México, D.F.: Universidad Autónoma Metropolitana; 2010.

6. Sung NS, Crowley Jr. WF, Genel M, Salber P, Sandy L, Sherwood LM, et al. Central challenges facing the national clinical research enterprise. J Am Med Assoc. 2003;289(10):1278-87.

7. Marincola FM. Translational medicine: a two-way road. J Transl Med. 2003;1:1.

8. National Center for Advancing Translational Sciences. Translational science spectrum factsheet. USA: NCATS; 2015. p. 1-2. https://ncats.nih.gov/files/translation-factsheet.pdf

9. Straus SE, Tetroe J, Graham I. Defining knowledge translation review. J Can Med Assoc. 2009;181(3-4):165-8. 
10. Graham I, Logan J, Harrison MB, Straus SE, Tetroe J, Caswell W, et al. Lost in knowledge map: time for a map? J Contin Educ Health Prof. 2006;26(1):13-24.

11. Graham I, Tetroe J. How to translate health research knowledge into effective healthcare action. Healthc Q. 2007;10(3):20-2.

12. Greenhalgh T, Wieringa S. Is it time to drop the "knowledge translation" metaphor? A critical literature review. J R Soc Med. 2011;104(12):501-9. Doi: 10.1258/jrsm.2011.110285

13. Estabrooks CA, Thompson DS, Lovely JJE, Hofmeyer A. A guide to knowledge translation theory. J Contin Educ Health Prof. 2006;26(1):25-36.

14. Davies H, Powell A, Nutley S. Mobilizing knowledge in health care. In: Ferlie E, Montgomery K, Reff Pedersen A, editors. Oxford handbook of health care management. Oxford University Press; 2016. p. 1-27. Doi: 10.1093/oxfordhb/9780198705109.013.17

15. Rabin BA, Brownson RC, Haire-Joshu D, Kreuter MW, Weaver NL. A glossary for dissemination and implementation research in health. J Public Heal Manag Pr. 2008;14(2):117-23.

16. Oborn E, Barrett M, Racko G. Knowledge translation in healthcare: a review of the literature. Cambridge, uk: Cambridge Judge Business School, University of Cambridge; 2010.

17. Nuyens Y, Lansang MAD. Knowledge translation: linking the past to the future. Bull World Health Organ. 2006;84(8):590-1.

18. World Health Organization. The Mexico statement on health research. Knowledge for better health: strengthening health systems. México: wно; 2004.

19. The World Health Organization. Bridging the "know-do" gap meeting on knowledge translation in global health. 2005.

20. Cabieses B, Bernales M, Obach A, Maturana A. El modelo de investigación traslacional: su aporte y desafíos para la toma de decisiones en salud. En: Llambias J (ed) La enfermedad de los sistemas de salud. Miradas críticas y alternativas. Santiago de Chile: RIL Editores; 2015. p. 99-124.

21. Cabieses B, Espinoza M. La investigación traslacional y su aporte para la toma de decisiones en políticas de salud. Rev Peru Med. 2011;28(2):288-97.

22. Lifshitz A. Medicina traslacional (traduccional, traducida, traslativa, trasladada). Med Int Méx. 2009;25(4):251-3.

23. Robles-Díaz G. Investigación traslacional. La Facultad de Medicina: un escenario. Gac Med Mex. 2011;147(3):288-92.

24. Valdespino-Gómez VM. La unidad de investigación traslacional como sustento de la medicina actual. Cir Cir. 2010;78(2):195-200.

25. Naidorf J, Alonso M. La movilización del conocimiento en tres tiempos. Rev Lusófona Educ [internet]. 2018;(39):81-95. Disponible en: http://revistas.ulusofona.pt/index.php/ rleducacao/article/view/6404

26. Goldblatt EM, Lee W-H. From bench to bedside: the growing use of translational research in cancer medicine. Am J Transl Res. 2010;2(1):1-18.

27. Drolet BC, Lorenzi NM. Translational research: understanding the continuum from bench to bedside. Transl Res. 2011;157(1):1-5. Doi: http://dx.doi.org/10.1016/j.trsl.2010.10.002 
28. Zerhouni E. Medicine. The NiH roadmap. Science. 2003;302(5642):63-72.

29. National Institutes of Health. A decade of discovery. The NiH roadmap and common fund. Vol. 15. National Institutes of Health; 2008. p. 59-60.

30. Woolf SH. The meaning of translational research and why it matters. JAMA. 2008;299(2):211-3.

31. Ogilvie D, Craig P, Griffin S, Macintyre S, Wareham NJ. A translational framework for public health research. BMc Public Health. 2009;9:116.

32. Westfall JM, Mold J, Fagnan L. Practice-based research- "blue highways" on the NiH roadmap. JAMA. 2007;297(4):403-6.

33. Ebener S, Khan A, Shademani R, Compernolle L, Beltran M, Lansang M, et al. Knowledge mapping as a technique to support knowledge translation. Bull World Health Organ. 2006;84:636-42.

34. Johnson LS. From knowledge transfer to knowledge translation: applying research to practice. Occup Ther Now. 2005;7(4):11-4.

35. Landry R, Amara N, Pablos-Mendes A, Shademani R, Gold I. The knowledge-value chain: a conceptual framework for knowledge translation in health. Bull World Health Organ. 2006;84(8):597-602.

36. Sur RL, Dahm P. History of evidence-based medicine. J Urol Soc IndiaI Ju. 2011;27(4):487-9.

37. Morris ZS, Wooding S, Grant J. The answer is 17 years, what is the question: understanding time lags in translational research. J R Soc Med. 2011;104(12):510-20.

38. Sudsawad P. Knowledge translation. Introduction to models, strategies, and measures [internet]. Vol. 2. Public Works. The National Center for the Dissemination of Disability Research, Southwest Educational Development Laboratory; 2007. p. 97-104. Available from: https://ktdrr.org/ktlibrary/articles_pubs/ktmodels/

39. Lomas J. La experiencia canadiense en la traducción del conocimiento: de la investigación a la práctica. En: Muñoz Hernández O, Durán Arenas L, editores. La traducción del conocimiento. Del resultado de la investigación a la aplicación en los servicios de salud. México: Instituto Mexicano del Seguro Social; 2006. p. 37-9.

40. Grimshaw JM, Eccles MP, Lavis JN, Hill SJ, Squires JE. Knowledge translation of research findings. Implement Sci. 2012;7(1):50. Doi: 10.1186/1748-5908-7-50

41. Rycroft-Malone J, Burton CR, Bucknall T, Graham ID, Hutchinson AM, Stacey D. Collaboration and co-production of knowledge in healthcare: opportunities and challenges. Kerman Univ Med Sci. 2016;5(x):1-3. Doi: http://dx.doi.org/10.15171/ijhpm.2016.08

42. Tricco AC, Cogo E, Ashoor H, Perrier L, McKibbon KA, Grimshaw JM, et al. Sustainability of knowledge translation interventions in healthcare decision-making: protocol for a scoping review. BMJ Open. 2013;3(5):1-3. Doi: 10.1186/s13012-016-0421-7

43. Field B, Booth A, Ilott I, Gerrish K. Using the knowledge to action framework in practice : a citation analysis and systematic review. Implement Sci. 2014;9(1):172-86. Doi: 10.1186/ s13012-014-0172-2

44. Levin, B. Thinking about knowledge mobilization: A discussion paper prepared at the request of the Canadian Council on Learning and the Social Sciences and Humanities Research Council. Toronto, Ontario: Ontario Institute for Studies in Education. 2008. 
http://www.sshrc-crsh.gc.ca/about-au_sujet/publications/KMb_-_LevinDiscussionPaper__E.pdf

45. De Greiff A, Maldonado O. “Apropiación fuerte” del conocimiento: una propuesta para construir políticas inclusivas de ciencia, tecnología e innovación en América Latina. En: Arellano A, Kreimer P, directores. Estudio social de la ciencia y la tecnología desde América Latina. Bogotá: Siglo del Hombre Editores; 2011. p. 209-62.

46. Olivé L. La apropiación social de la ciencia y la tecnología. En: Pérez-Bustos T, Lozano Borda M, editors. Ciencia, tecnología y democracia: reflexiones en torno a la apropiación social del conocimiento. Medellín: Universidad Eafit-Colciencias; 2011. p. 114-21.

47. Walshe K, Davies HT. Health research, development and innovation in England from 1988 to 2013: from research production to knowledge mobilization. J Health Serv Res Policy. 2013;18(3):1-12. Doi: 10.1177/1355819613502011

48. Cooksey, D. A review of UK health research funding. London, UK: The Stationery Office; 2006. p. 1-120. https://assets.publishing.service.gov.uk/government/uploads/system/ uploads/attachment_data/file/228984/0118404881.pdf

49. Báscolo E, Yavich N, Sánchez de León A, De León AS. El proceso de interacción investigadores y tomadores de decisiones: un estudio de caso. Cad Saude Publica. 2006;22 sup:S47-56.

50. Lavis JN. Research, public policymaking, and knowledge-translation processes: Canadian efforts to build bridges. J Contin Educ Health Prof. 2006;26(1):37-45.

51. Muñoz Hernández O, Durán Arenas L. El Instituto Mexicano del Seguro Social: establecimiento de un puente entre la investigación y la práctica en los servicios de salud. En: Muñoz Hernández O, Durán Arenas L, editores. La traducción del conocimiento. Del resultado de la investigación a la aplicación en los servicios de salud. México: Instituto Mexicano del Seguro Social; 2006. p. 15-24.

52. Lavis JN, Lomas J, Hamid M, Sewankambo NK. Assessing country-level efforts to link research to action. Bull World Health Organ. 2006;84(8):620-8.

53. Rajan A, Sullivan R, Bakker S, Van Harten WH. Critical appraisal of translational research models for suitability in performance assessment of cancer centers. Oncologist. 2012;17(12):e48-57. Doi: 10.1634/theoncologist.2012-0216

54. Barrios Suarez E, Logie C, Arocha JF. An open debate about the object and purpose of global health knowledge in the context of an interdisciplinary research partnership on HIV/ Sті prevention priorities in Peru. Global Health. 2014;10:40. Doi: 10.1186/1744-8603-10-40

55. Natera JM, Rojas-Rajs S, Dutrénit G, Vera-Cruz AO. National health problems and useful knowledge: lessons from public funding of diabetes research in Mexico. Innov Dev. 2019. 9(2):205-224 Doi: 10.1080/2157930X.2019.1567906 